\title{
ON FOURIER TRANSFORM OF A CLASS OF ENTIRE FUNCTIONS
}

\author{
I.KH. MUSIN, M.I. MUSIN
}

\begin{abstract}
We consider a space of entire functions of several complex variables decaying fast on $\mathbb{R}^{n}$ and such that their growth along $i \mathbb{R}^{n}$ is majorized by means of a family of weight functions. Under certain assumptions for the weight functions we obtain an equivalent description of this space in terms of estimates for partial derivatives of the functions in $\mathbb{R}^{n}$ and prove a Paley-Wiener type theorem.
\end{abstract}

Keywords: Gelfand-Shilov spaces, Fourier transform, entire functions, convex functions.

Mathematics Subject Classification: 32A15, 42B10, 46E10, 46F05, 42A38

\section{INTRODUCTION}

1.1. On a problem. Let $\Phi=\left\{\varphi_{m}\right\}_{m=1}^{\infty}$ be a family of non-decreasing functions $\varphi_{m}$ : $[0, \infty) \rightarrow \mathbb{R}$ such that for each $m \in \mathbb{N}$ :

$\left.i_{1}\right) . \lim _{x \rightarrow+\infty} \frac{\varphi_{m}(x)}{x}=+\infty$;

$\left.i_{2}\right)$. for each $A>0$ there exists a constant $C(m, A)>0$ such that

$$
\varphi_{m}(x)+A \ln (1+x) \leqslant \varphi_{m+1}(x)+C(m, A), x \geqslant 0 .
$$

Let $H\left(\mathbb{C}^{n}\right)$ be a space of entire functions in $\mathbb{C}^{n}$ with the usual topology. Given $u \in \mathbb{R}^{n}\left(\mathbb{C}^{n}\right)$, by $\|u\|$ we denote its Euclidean norm.

For arbitrary $\nu \in \mathbb{N}$ and $k \in \mathbb{Z}_{+}$we introduce the normed space

$$
E_{k}\left(\varphi_{\nu}\right)=\left\{f \in H\left(\mathbb{C}^{n}\right): p_{\nu, k}(f)=\sup _{z \in \mathbb{C}^{n}} \frac{\|f(z)\|(1+\|z\|)^{k}}{e^{\varphi_{\nu}}(\|\operatorname{Im} z\|)}<\infty\right\} .
$$

Let $E\left(\varphi_{\nu}\right)$ be the projective limit of spaces $E_{k}\left(\varphi_{\nu}\right), E(\Phi)$ be the inductive limit of spaces $E\left(\varphi_{\nu}\right)$.

We note that if we define function $\varphi_{\nu}$ by the formula $\varphi_{\nu}(x)=\Omega(\nu x)(\nu \in \mathbb{N})$, where $\Omega$ is a real-valued continuously differentiable function on $[0, \infty)$ such that $\Omega(0)=\Omega^{\prime}(0)=0, \Omega^{\prime}$ increases, $\lim _{x \rightarrow+\infty} \Omega^{\prime}(x)=+\infty$ (thus, in this case the weight functions $\varphi_{\nu}(\|x\|)$ are convex in $\mathbb{R}^{n}$ ), then $E(\Phi)$ coincides with Gelfand-Shilov space $W^{\Omega}$ [1] [5]. In works [1]-[5], the Fourier transform of space $W^{\Omega}$ was studied and an alternative definition of $W^{\Omega}$ was given. We note that in work [6], while studying similar issues in a close to $W^{\Omega}$ (by the matter) space of entire functions decaying fast on the real axis, the authors succeeded to get rid of the convexity of weight functions.

The aim of the work is to describe the Fourier transform of space $E(\Phi)$ and to characterizes $E(\Phi)$ in terms of estimates for partial derivatives of functions in $\mathbb{R}^{n}$ for rather general additional conditions for $\Phi$ extending the approach of work [6] for the case of many variables.

1.2. Notations and definitions. For $u=\left(u_{1}, \ldots, u_{n}\right), v=\left(v_{1}, \ldots, v_{n}\right) \in \mathbb{R}^{n}\left(\mathbb{C}^{n}\right)$ we let $\langle u, v\rangle=u_{1} v_{1}+\cdots+u_{n} v_{n}$.

I.Kh. Musin, M.I. Musin, On Fourier transform of a Class of Entire functions.

(c) Musin I.Kh., Musin M.I. 2014.

The work of the first author was supported by Russian Foundation for Basic Researches (grants nos. 14-0100720, 14-01-97037) and Program of BMS RAS.

Submitted November 5, 2014. 
For $\alpha=\left(\alpha_{1}, \ldots, \alpha_{n}\right) \in \mathbb{Z}_{+}^{n}, x=\left(x_{1}, \ldots, x_{n}\right) \in \mathbb{R}^{n}, z=\left(z_{1}, \ldots, z_{n}\right) \in \mathbb{C}^{n},\|\alpha\|=\alpha_{1}+\ldots+\alpha_{n}$, $x^{\alpha}=x_{1}^{\alpha_{1}} \cdot \ldots \cdot x_{n}^{\alpha_{n}}, z^{\alpha}=z_{1}^{\alpha_{1}} \cdot \ldots \cdot z_{n}^{\alpha_{n}}, D^{\alpha}=\frac{\partial^{\|\alpha\|}}{\partial x_{1}^{\alpha_{1}} \ldots \partial x_{n}^{\alpha_{n}}}$.

If $\alpha=\left(\alpha_{1}, \ldots, \alpha_{n}\right), \beta=\left(\beta_{1}, \ldots, \beta_{n}\right) \in \mathbb{Z}_{+}^{n}$, then the notation $\alpha \leqslant \beta$ indicates that $\alpha_{j} \leqslant \beta_{j}$ $(j=1,2, \ldots, n)$; as $\alpha \leqslant \beta$ we let $C_{\beta}^{\alpha}=\prod_{j=1}^{n} C_{\beta_{j}}^{\alpha_{j}}$.

$s_{n}(1)$ stands for the area of the unit sphere in $\mathbb{R}^{n}$.

Given a function $u:[0, \infty) \rightarrow \mathbb{R}$, we let $u[e](x):=u\left(e^{x}\right), x \geqslant 0$.

For the sake of brevity we denote $\varphi_{m}[e]$ by $\psi_{m}(m \in \mathbb{N})$.

$\mathcal{B}$ is the set of all continuous functions $g:[0, \infty) \rightarrow \mathbb{R}$ satisfying the identity $\lim _{x \rightarrow+\infty} \frac{g(x)}{x}=+\infty$.

Let $V=\{h \in \mathcal{B}: h$ is convex on $[0, \infty)\}, \mathcal{V}=\{h \in V: h$ grows on $[0, \infty)$ and $h(0)=0\}$.

For $g \in V$ we let $V_{g}=\left\{h \in \mathcal{V}: h\right.$ coincides with $g$ on $\left[d_{h}, \infty\right)$, where $d_{h}$ is a positive number depending on $h\}$.

The Young transform $g^{*}$ of function $g \in \mathcal{B}$ is introduced by the formula $g^{*}(x)=\sup _{y \geqslant 0}(x y-$ $g(y)), x \geqslant 0$.

1.3. Main results. Let $\Psi^{*}=\left\{\psi_{\nu}^{*}\right\}_{\nu=1}^{\infty}$. Given $\nu \in \mathbb{N}$ and $m \in \mathbb{Z}_{+}$, we let

$$
\mathcal{E}_{m}\left(\psi_{\nu}^{*}\right)=\left\{f \in C^{\infty}\left(\mathbb{R}^{n}\right): \mathcal{R}_{m, \nu}(f)=\sup _{x \in \mathbb{R}^{n}, \alpha \in \mathbb{Z}_{+}^{n}} \frac{(1+\|x\|)^{m}\left\|\left(D^{\alpha} f\right)(x)\right\|}{\alpha ! e^{-\psi_{\nu}^{*}(\|\alpha\|)}}<\infty\right\} .
$$

Let $\mathcal{E}\left(\psi_{\nu}^{*}\right)=\bigcap_{m=0}^{\infty} \mathcal{E}_{m}\left(\psi_{\nu}^{*}\right), \mathcal{E}\left(\Psi^{*}\right)=\bigcup_{\nu=1}^{\infty} \mathcal{E}\left(\psi_{\nu}^{*}\right)$.

Theorems 1 and 2 proven in Section 3 by standard schemes are aimed for describing functions in $E(\Phi)$ in terms of estimates for partial derivatives in $\mathbb{R}^{n}$.

Theorem 1. Suppose that family $\Phi$ satisfies condition

$\left.i_{3}\right)$. for each $m \in \mathbb{N}$ there exists a constant $a_{m}>0$ such that

$$
\varphi_{m}(2 x) \leqslant \varphi_{m+1}(x)+a_{m}, x \geqslant 0 .
$$

If $f \in E(\Phi)$, then $f_{\mid \mathbb{R}^{n}} \in \mathcal{E}\left(\Psi^{*}\right)$.

Theorem 2. Suppose that family $\Phi$ satisfies condition

$\left.i_{3}^{\prime}\right)$. for each $m \in \mathbb{N}$ there exist constants $\sigma_{m}>1$ and $\gamma_{m}>0$ such that

$$
\varphi_{m}\left(\sigma_{m} x\right) \leqslant \varphi_{m+1}(x)+\gamma_{m}, x \geqslant 0 .
$$

Then each function $f \in \mathcal{E}\left(\Psi^{*}\right)$ has the unique continuation to an entire function belonging to $E(\Phi)$.

The proofs of Theorems 1 and 2 provide additional information on the structure of $E(\Phi)$. Namely, given $\nu \in \mathbb{N}$ let $\mathcal{H}\left(\varphi_{\nu}\right)$ be the projective limit of the spaces

$$
\mathcal{H}_{k}\left(\varphi_{\nu}\right)=\left\{f \in H\left(\mathbb{C}^{n}\right): \mathcal{N}_{\nu, k}(f)=\sup _{z \in \mathbb{C}^{n}} \frac{\|f(z)\|(1+\|z\|)^{k}}{e^{\left(\psi_{\nu}^{*}\right)^{*}(\ln (1+\|\operatorname{Im} z\|))}}<\infty\right\}, \quad k \in \mathbb{Z}_{+} .
$$

Let $\mathcal{H}(\Phi)$ be the inductive limit of spaces $\mathcal{H}\left(\varphi_{\nu}\right)$. It will be shown in Section 3 that if family $\Phi$ satisfies condition $i_{3}$ ), then $E(\Phi)=\mathcal{H}(\Phi)$ (see Proposition 1).

Passing to problems related with Fourier transform in $E(\Phi)$, we introduce one more class of infinitely differentiable functions in $\mathbb{R}^{n}$. Let $U=\left\{u_{\nu}\right\}_{\nu=1}^{\infty}$ be an arbitrary family of nondecreasing convex functions $u_{\nu}$ on $[0, \infty)$ such that for each $\nu \in \mathbb{N}$ :

$\left.i_{1}\right) \cdot \lim _{x \rightarrow+\infty} \frac{u_{\nu}(x)}{x}=+\infty$;

$\left.i_{2}\right) . \lim _{x \rightarrow+\infty}\left(u_{\nu}(x)-u_{\nu+1}(x)\right)=+\infty$. 
Given $\nu \in \mathbb{N}$ and $m \in \mathbb{Z}_{+}$, let

$$
G_{m}\left(u_{\nu}\right)=\left\{f \in C^{m}\left(\mathbb{R}^{n}\right):\|f\|_{m, u_{\nu}}=\sup _{\substack{x \in \mathbb{R}^{n},\|\alpha\| \leqslant m, \beta \in \mathbb{Z}_{+}^{n}}} \frac{\left\|x^{\beta}\left(D^{\alpha} f\right)(x)\right\|}{\|\beta\| ! e^{-u_{\nu}(\|\beta\|)}}<\infty\right\} .
$$

Let $G\left(u_{\nu}\right)=\bigcap_{m=0}^{\infty} G_{m}\left(u_{\nu}\right), G(U)=\bigcup_{\nu=1}^{\infty} G\left(u_{\nu}\right)$. We equip $G\left(u_{\nu}\right)$ by the topology induced the family of the norms $\|\cdot\|_{m, u_{\nu}}\left(m \in \mathbb{Z}_{+}\right)$, and $G(U)$ is equipped by the topology of the inductive limit of the spaces $G\left(u_{\nu}\right)$.

We introduce Fourier transform $\hat{f}$ of a function $f \in E(\Phi)$ by the formula

$$
\hat{f}(x)=\int_{\mathbb{R}^{n}} f(\xi) e^{-i\langle x, \xi\rangle} d \xi, x \in \mathbb{R}^{n} .
$$

In Section 4 we shall prove

Theorem 3. Suppose that $\Phi$ satisfies condition $\left.i_{3}\right)$ of Theorem 1 and condition

$\left.i_{4}\right)$. for each $m \in \mathbb{N}$ there exist constants $h_{m}>1$ and $l_{m}>0$ such that

$$
2 \varphi_{m}(x) \leqslant \varphi_{m+1}\left(h_{m} x\right)+l_{m}, x \geqslant 0 .
$$

Then Fourier transform $\mathcal{F}: f \in E(\Phi) \rightarrow \hat{f}$ makes an isomorphism between spaces $E(\Phi)$ and $G\left(\Psi^{*}\right)$.

Let $\Phi^{*}=\left\{\varphi_{\nu}^{*}\right\}_{\nu=1}^{\infty}$. Given $\nu \in \mathbb{N}$ and $m \in \mathbb{Z}_{+}$, we let

$$
G S_{m}\left(\varphi_{\nu}^{*}\right)=\left\{f \in C^{m}\left(\mathbb{R}^{n}\right): q_{m, \nu}(f)=\sup _{x \in \mathbb{R}^{n},\|\alpha\| \leqslant m} \frac{\left\|\left(D^{\alpha} f\right)(x)\right\|}{e^{-\varphi_{\nu}^{*}(\|x\|)}}<\infty\right\} .
$$

For $\nu \in \mathbb{N}$ we let $G S\left(\varphi_{\nu}^{*}\right)=\bigcap_{m \in \mathbb{Z}_{+}} G S_{m}\left(\varphi_{\nu}^{*}\right)$. We denote $G S\left(\Phi^{*}\right)=\bigcup_{\nu \in \mathbb{N}} G S\left(\varphi_{\nu}^{*}\right)$. We equip $G S\left(\varphi_{\nu}^{*}\right)$ by the topology induced by the system of norms $q_{\nu, m}\left(m \in \mathbb{Z}_{+}\right)$, and $G S\left(\Phi^{*}\right)$ is equipped by the topology of inductive limit of spaces $G S\left(\varphi_{\nu}^{*}\right)$.

In the fifth section we shall prove the following theorem.

Theorem 4. Suppose that family $\Phi$ consists of convex functions and satisfies condition $\left.i_{3}\right)$ of Theorem 1. Then $G\left(\Psi^{*}\right)=G S\left(\Phi^{*}\right)$.

\section{AuXiLiary Results}

In the proof of the theorems we shall make use of the following three lemmata.

Lemma 1. Let $g \in \mathcal{B}$. Then for each $M>0$ there exists a constant $A_{M}>0$ such that

$$
(g[e])^{*}(x) \leqslant x \ln \frac{x}{M}-x+A_{M}
$$

for each $x>0$.

This lemma was proven in [6].

Corollary 1. Let $g \in \mathcal{B}$. Then for each $b>0$ the series $\sum_{\alpha \in \mathbb{Z}_{+}^{n}} \frac{e^{(g[e])^{*}(\|\alpha\|)}}{b^{\|\alpha\|}\|\alpha\| !}$ converges.

In the same way as Lemma 2 in [6] we prove the following lemma.

Lemma 2. Suppose that $u, v \in \mathcal{B}$ and there exist numbers $\tau>0$ and $C>0$ such that

$$
2 u(x) \leqslant v(x+\tau)+C, x \geqslant 0 .
$$

Then there exist a number $A>0$ such that

$$
v^{*}(x+y) \leqslant u^{*}(x)+u^{*}(y)+\tau(x+y)+A, x, y \geqslant 0 .
$$


Lemma 3. Suppose that real-valued functions $u, v \in C[0, \infty)$ be so that

1. $\lim _{x \rightarrow+\infty} \frac{u[e](x)}{x}=\lim _{x \rightarrow+\infty} \frac{v[e](x)}{x}=+\infty$;

2. there exist numbers $\sigma>1$ and $\gamma>0$ such that

$$
u(\sigma x) \leqslant v(x)+\gamma, x \geqslant 0 .
$$

Then

$$
(u[e])^{*}(x)-(v[e])^{*}(x) \geqslant x \ln \sigma-\gamma, x \geqslant 0 .
$$

Proof. It is clear that $u[e](t+\ln \sigma) \leqslant v[e](t)+\gamma, t \geqslant 0$. Then for each $x \geqslant 0$

$$
\begin{aligned}
(u[e])^{*}(x)-(v[e])^{*}(x) & =\sup _{t \geqslant 0}(x t-u[e](t))-\sup _{t \geqslant 0}(x t-v[e](t)) \\
& \geqslant \sup _{t \geqslant 0}(x t-u[e](t))-\sup _{t \geqslant 0}(x t-u[e](t+\ln \sigma))-\gamma \\
& =\sup _{t \geqslant 0}(x t-u[e](t))-\sup _{t \geqslant 0}(x(t+\ln \sigma)-u[e](t+\ln \sigma))+x \ln \sigma-\gamma \\
& \geqslant x \ln \sigma-\gamma .
\end{aligned}
$$

3. Equivalent DESCRIPTION OF $E(\Phi)$ Under ADDitional CONDitions For $\Phi$

3.1. Proof of Theorem 1. Let $f \in E(\Phi)$. Then $f \in E\left(\varphi_{\nu}\right)$ for some $\nu \in \mathbb{N}$. Let $m \in \mathbb{Z}_{+}$, $\alpha \in \mathbb{Z}_{+}^{n}$ and $x \in \mathbb{R}^{n}$ be arbitrary. By integral Cauchy formula we get

$$
(1+\|x\|)^{m}\left(D^{\alpha} f\right)(x)=\frac{\alpha !}{(2 \pi i)^{n}} \int_{L_{R}(x)} \ldots \int \frac{f(\zeta)(1+\|x\|)^{m} d \zeta}{\left(\zeta_{1}-x_{1}\right)^{\alpha_{1}+1} \cdots\left(\zeta_{n}-x_{n}\right)^{\alpha_{n}+1}},
$$

where $L_{R}(x)=\left\{\zeta=\left(\zeta_{1}, \ldots, \zeta_{n}\right) \in \mathbb{C}^{n}:\left\|\zeta_{j}-x_{j}\right\|=R, j=1, \ldots, n\right\}$ for each $R>0$. It implies

$$
\begin{aligned}
(1+\|x\|)^{m}\left\|\left(D^{\alpha} f\right)(x)\right\| & \leqslant \frac{\alpha !}{(2 \pi)^{n}} \int \ldots \int \frac{(1+\|x-\zeta\|)^{m}(1+\|\zeta\|)^{m}\|f(\zeta)\| d \zeta \|}{\left\|\zeta_{1}-x_{1}\right\|^{\alpha_{1}+1} \cdots\left\|\zeta_{n}-x_{n}\right\|^{\alpha_{n}+1}} \\
& \leqslant \frac{\alpha ! p_{\nu, m}(f)(1+n R)^{m} e^{\varphi_{\nu}(n R)}}{R^{\|\alpha\|}} .
\end{aligned}
$$

Employing condition $i_{2}$ ) for $\Phi$ we obtain that

$$
(1+\|x\|)^{m}\left\|\left(D^{\alpha} f\right)(x)\right\| \leqslant e^{C(\nu, m)} \alpha ! p_{\nu, m}(f) \frac{e^{\varphi_{\nu+1}(n R)}}{R^{\|\alpha\|}} .
$$

Therefore,

$$
\begin{aligned}
& (1+\|x\|)^{m}\left\|\left(D^{\alpha} f\right)(x)\right\| \leqslant e^{C(\nu, m)} n^{\|\alpha\|} \alpha ! p_{\nu, m}(f) \inf _{R>0} \frac{e^{\varphi_{\nu+1}(R)}}{R^{\|\alpha\|}} \\
& \leqslant e^{C(\nu, m)} n^{\|\alpha\|} \alpha ! p_{\nu, m}(f) \exp \left(-\sup _{R>1}\left(\|\alpha\| \ln R-\varphi_{\nu+1}(R)\right)\right. \\
& =e^{C(\nu, m)} n^{\|\alpha\|} \alpha ! p_{\nu, m}(f) e^{-\sup _{r>0}\left(\|\alpha\| r-\psi_{\nu+1}(r)\right)}=e^{C(\nu, m)} n^{\|\alpha\|} \alpha ! p_{\nu, m}(f) e^{-\psi_{\nu+1}^{*}(\|\alpha\|)} .
\end{aligned}
$$

Since by condition $i_{3}$ ) and Lemma 3

$$
\psi_{k}^{*}(x)-\psi_{k+1}^{*}(x) \geqslant x \ln 2-a_{k}, x \geqslant 0,
$$

for $k \in \mathbb{N}$, employing inequality (1) we obtain

$$
(1+\|x\|)^{m}\left\|\left(D^{\alpha} f\right)(x)\right\| \leqslant a_{\nu, m} p_{\nu, m}(f) \alpha ! e^{-\psi_{\nu+n}^{*}(\|\alpha\|)}, x \in \mathbb{R}^{n}, \alpha \in \mathbb{Z}_{+}^{n},
$$

where $a_{\nu, m}$ is a positive number depending on $\nu$ and $m$. Thus, for each $m \in \mathbb{Z}_{+}$

$$
\mathcal{R}_{m, \nu+n}\left(f_{\mid \mathbb{R}^{n}}\right) \leqslant a_{\nu, m} p_{\nu, m}(f) .
$$


Hence, $f_{\mid \mathbb{R}^{n}} \in \mathcal{E}\left(\psi_{\nu+n}^{*}\right)$. Therefore, $f_{\mid \mathbb{R}^{n}} \in \mathcal{E}\left(\Psi^{*}\right)$.

3.2. Proof of Theorem 2. Let $f \in \mathcal{E}\left(\Psi^{*}\right)$. Then $f \in \mathcal{E}\left(\psi_{\nu}^{*}\right)$ for some $\nu \in \mathbb{N}$. Therefore, for each $m \in \mathbb{Z}_{+}$we have

$$
(1+\|x\|)^{m}\left\|\left(D^{\alpha} f\right)(x)\right\| \leqslant \mathcal{R}_{m, \nu}(f) \alpha ! e^{-\psi_{\nu}^{*}(\|\alpha\|)}, x \in \mathbb{R}^{n}, \alpha \in \mathbb{Z}_{+}^{n} .
$$

Since $\lim _{x \rightarrow+\infty} \frac{\psi_{\nu}^{*}(x)}{x}=+\infty$, inequality (3) yields that for each $\varepsilon>0$ there exists a number $c_{\varepsilon}>0$ such that $\left\|\left(D^{\alpha} f\right)(x)\right\| \leqslant c_{\varepsilon} \varepsilon^{\|\alpha\|} \alpha$ !. for each $x \in \mathbb{R}^{n}$ and $\alpha \in \mathbb{Z}_{+}^{n}$ It is clear that the subsequence $\left(\sum_{\|\alpha\| \leqslant k} \frac{\left(D^{\alpha} f\right)(0)}{\alpha !} x^{\alpha}\right)_{k=1}^{\infty}$ converges to $f$ uniformly on compact sets $\mathbb{R}^{n}$ and the series $\sum_{\|\alpha\| \geqslant 0} \frac{\left(D^{\alpha} f\right)(0)}{\alpha !} z^{\alpha}$ converges in $H\left(\mathbb{C}^{n}\right)$ and its sum $F_{f}(z)$ is an entire function. We observe that $F_{f \mid \mathbb{R}^{n}}=f$. The uniqueness of the holomorphic continuation is obvious.

Let us show that $F_{f} \in E(\Phi)$. We estimate the growth of $F_{f}$ by employing inequality (3) and the expansion of $F_{f}$ into the Taylor series at point $x \in \mathbb{R}^{n}$ :

$$
F_{f}(z)=\sum_{\|\alpha\| \geqslant 0} \frac{\left(D^{\alpha} f\right)(x)}{\alpha !}(i y)^{\alpha}, \quad z=x+i y, \quad y \in \mathbb{R}^{n} .
$$

Let $m \in \mathbb{Z}_{+}$be arbitrary. Then

$$
\begin{aligned}
(1+\|z\|)^{m}\left\|F_{f}(z)\right\| & \leqslant \sum_{\|\alpha\| \geqslant 0} \frac{(1+\|x\|)^{m}(1+\|y\|)^{m+\|\alpha\|}\left\|\left(D^{\alpha} f\right)(x)\right\|}{\alpha !} \\
& \leqslant \sum_{\|\alpha\| \geqslant 0} \mathcal{R}_{m, \nu}(f) e^{-\psi_{\nu}^{*}(\|\alpha\|)}(1+\|y\|)^{m+\|\alpha\|} \\
& \leqslant \mathcal{R}_{m, \nu}(f)(1+\|y\|)^{m} \sum_{\|\alpha\| \geqslant 0} \frac{(1+\|y\|)^{\|\alpha\|}}{e^{\psi_{\nu+1}^{*}(\|\alpha\|)}} e^{\psi_{\nu+1}^{*}(\|\alpha\|)-\psi_{\nu}^{*}(\|\alpha\|)} .
\end{aligned}
$$

Employing that by Lemma 3

$$
\psi_{k}^{*}(x)-\psi_{k+1}^{*}(x) \geqslant \delta_{k} x-\gamma_{k}, x \geqslant 0,
$$

where $\delta_{k}=\ln \sigma_{k}$, and denoting $\left(\frac{e^{\gamma_{\nu}+\delta_{\nu}}}{e^{\delta_{\nu}-1}}\right)^{n}$ by $B_{\nu}$, we obtain

$$
(1+\|z\|)^{m}\left\|F_{f}(z)\right\| \leqslant B_{\nu} \mathcal{R}_{m, \nu}(f)(1+\|y\|)^{m} e^{\sup ^{t \geqslant 0}\left(t \ln (1+\|y\|)-\psi_{\nu+1}^{*}(t)\right)} .
$$

Hence,

$$
(1+\|z\|)^{m}\left\|F_{f}(z)\right\| \leqslant B_{\nu} \mathcal{R}_{m, \nu}(f) e^{\left.\left(\psi_{\nu+1}^{*}\right)^{*}(\ln (1+\|y\|))+m \ln (1+\|y\|)\right)} .
$$

We note that condition $i_{2}$ ) yields that for each $k \in \mathbb{N}$ and $A>0$

$$
\psi_{k}(x)+A x \leqslant \psi_{k+1}(x)+C(k, A), \quad x \geqslant 0 .
$$

It implies immediately that for each $\xi \geqslant 0$

$$
\psi_{k}^{*}(\xi) \geqslant \psi_{k+1}^{*}(\xi+A)-C(k, A) .
$$

Then

$$
\begin{aligned}
\left(\psi_{k}^{*}\right)^{*}(x) & =\sup _{\xi \geqslant 0}\left(x \xi-\psi_{k}^{*}(\xi)\right) \leqslant \sup _{\xi \geqslant 0}\left(x \xi-\psi_{k+1}^{*}(\xi+A)\right)+C(k, A) \\
& =\sup _{\xi \geqslant 0}\left(x(\xi+A)-\psi_{k+1}^{*}(\xi+A)\right)-A x+C(k, A) \leqslant\left(\psi_{k+1}^{*}\right)^{*}(x)-A x+C(k, A)
\end{aligned}
$$

for each $x \geqslant 0$. Thus, for each $k \in \mathbb{N}$ and $A>0$ we have

$$
\left(\psi_{k}^{*}\right)^{*}(x)+A x \leqslant\left(\psi_{k+1}^{*}\right)^{*}(x)+C(k, A), x \geqslant 0 .
$$

Employing now inequality (6), by estimate (5) we obtain

$$
(1+\|z\|)^{m}\left\|F_{f}(z)\right\| \leqslant B_{\nu} \mathcal{R}_{m, \nu}(f) e^{C_{\nu+1, m}} e^{\left(\psi_{\nu+2}^{*}\right)^{*}(\ln (1+\|y\|))} .
$$


It is clear that

$$
(1+\|z\|)^{m}\left\|F_{f}(z)\right\| \leqslant B_{\nu} \mathcal{R}_{m, \nu}(f) e^{C_{\nu+1, m}} e^{\psi_{\nu+2}(\ln (1+\|y\|))} .
$$

It means that

$$
(1+\|z\|)^{m}\left\|F_{f}(z)\right\| \leqslant B_{\nu} \mathcal{R}_{m, \nu}(f) e^{C_{\nu+1, m}} e^{\varphi_{\nu+2}(1+\|y\|)} .
$$

Employing the non-decreasing of functions family $\Phi$ and condition $i_{3}^{\prime}$ ), we find a constant $K_{\nu, m}>$ 0 such that

for each $z \in \mathbb{C}^{n}$.

$$
(1+\|z\|)^{m}\left\|F_{f}(z)\right\| \leqslant K_{\nu, m} \mathcal{R}_{m, \nu}(f) e^{\varphi_{\nu+3}(\|\operatorname{Im} z\|)}
$$

Thus, for each $m \in \mathbb{Z}_{+}, p_{\nu+3, m}\left(F_{f}\right) \leqslant K_{\nu, m} \mathcal{R}_{m, \nu}(f)$. Therefore, $F_{f} \in E\left(\varphi_{\nu+3}\right)$. Hence, $F_{f} \in E(\Phi)$. The proof is complete.

3.3. Remark on space $E(\Phi)$. We recall that in the first section we have introduced spaces $\mathcal{H}_{k}\left(\varphi_{\nu}\right), \mathcal{H}\left(\varphi_{\nu}\right)$, and $\mathcal{H}(\Phi)$ as follows. Given arbitrary $\nu \in \mathbb{N}$ and $k \in \mathbb{Z}_{+}$, let

$$
\mathcal{H}_{k}\left(\varphi_{\nu}\right)=\left\{f \in H\left(\mathbb{C}^{n}\right): \mathcal{N}_{\nu, k}(f)=\sup _{z \in \mathbb{C}^{n}} \frac{\|f(z)\|(1+\|z\|)^{k}}{e^{\left(\psi_{\nu}^{*}\right)^{*}(\ln (1+\|\operatorname{Im} z\|))}}<\infty\right\} .
$$

Let $\mathcal{H}\left(\varphi_{\nu}\right)=\bigcap_{k=0}^{\infty} \mathcal{H}_{k}\left(\varphi_{\nu}\right), \mathcal{H}(\Phi)=\bigcup_{\nu=1}^{\infty} \mathcal{H}\left(\varphi_{\nu}\right)$. Since for $f \in \mathcal{H}_{k+1}\left(\varphi_{\nu}\right)$ we have $\mathcal{N}_{\nu, k}(f) \leqslant$ $\mathcal{N}_{\nu, k+1}(f)$, then $\mathcal{H}_{k+1}\left(\varphi_{\nu}\right)$ is continuously embedded into $\mathcal{H}_{k}\left(\varphi_{\nu}\right)$. We equip $\mathcal{H}\left(\varphi_{\nu}\right)$ by the topology induced by the family of norms $\mathcal{H}_{k}\left(\varphi_{\nu}\right)$. In view of inequality (6), we see that if $f \in \mathcal{H}\left(\varphi_{\nu}\right)$, then $\mathcal{N}_{\nu+1, k}(f) \leqslant e^{C(\nu, 1)} \mathcal{N}_{\nu, k}(f)$ for each $k \in \mathbb{Z}_{+}$. Thus, $\mathcal{H}\left(\varphi_{\nu}\right)$ is continuously embedded into $\mathcal{H}\left(\varphi_{\nu+1}\right)$ for each $\nu \in \mathbb{N}$. We equip $\mathcal{H}(\Phi)$ by the topology of the inductive limit of spaces $\mathcal{H}\left(\varphi_{\nu}\right)$.

Proposition 1. Suppose that family $\Phi$ satisfies condition $\left.i_{3}\right)$. Then $E(\Phi)=\mathcal{H}(\Phi)$.

Proof. Let us show first that $\mathcal{H}(\Phi)$ is continuously embedded into $E(\Phi)$. Let $\nu \in \mathbb{N}$ and $f \in$ $\mathcal{H}\left(\varphi_{\nu}\right)$. Employing the non-decreasing of $\varphi_{\nu}$ and condition $\left.i_{3}\right)$ for $\Phi$, we find a constant $K_{\nu}>0$ such that for each $k \in \mathbb{Z}_{+}$

$$
p_{\nu+1, k}(f) \leqslant K_{\nu} \mathcal{N}_{\nu, k}(f), f \in \mathcal{H}\left(\varphi_{\nu}\right) .
$$

It follows that $f \in E(\Phi)$ and the embedding $I: \mathcal{H}(\Phi) \rightarrow E(\Phi)$ is continuous.

Let us show that mapping $I$ is surjective. Let $f \in E(\Phi)$. Then $f \in E\left(\varphi_{\nu}\right)$ for some $\nu \in \mathbb{N}$. Let $m \in \mathbb{Z}_{+}$be arbitrary. We recall that by inequality $(2) \mathcal{R}_{m, \nu+n}\left(f_{\mid \mathbb{R}}\right) \leqslant a_{\nu, m} p_{\nu, m}(f)$. Together with inequality (7) (with $\nu$ replaced by $\nu+n$; we also recall that in our case $\sigma=2$ for each $m \in \mathbb{N})$ it implies that

$$
\mathcal{N}_{\nu+n+2, m}(f) \leqslant A_{\nu, m} p_{\nu, m}(f),
$$

where $A_{\nu, m}>0$ is a constant. Thus, $f \in \mathcal{H}\left(\varphi_{\nu+n+2}\right)$. Therefore, $f \in \mathcal{H}(\Phi)$. Moreover, the latter estimate shows that the inverse mapping $I^{-1}$ is continuous. Hence, $E(\Phi)=\mathcal{H}(\Phi)$.

\section{Fourier tRANSFORM IN $E(\Phi)$}

4.1. Simpler description of space $G\left(\Psi^{*}\right)$. Let us show that if $\Phi$ satisfies condition $\left.i_{3}\right)$, then space $G\left(\Psi^{*}\right)$ admits a simpler description. In order to do it, we introduce space $Q\left(\Psi^{*}\right)$ as follows. For $\nu \in \mathbb{N}$ and $m \in \mathbb{Z}_{+}$let

$$
Q_{m}\left(\psi_{\nu}^{*}\right)=\left\{f \in C^{m}\left(\mathbb{R}^{n}\right): N_{\nu, m}(f)=\max _{\|\alpha\| \leqslant m} \sup _{x \in \mathbb{R}^{n}, k \in \mathbb{Z}_{+}} \frac{(1+\|x\|)^{k}\left\|\left(D^{\alpha} f\right)(x)\right\|}{k ! e^{-\psi_{\nu}^{*}(k)}}<\infty\right\} .
$$

Let $Q\left(\psi_{\nu}^{*}\right)=\bigcap_{m \in \mathbb{Z}_{+}} Q_{m}\left(\psi_{\nu}^{*}\right), Q\left(\Psi^{*}\right)=\bigcup_{\nu \in \mathbb{N}} Q\left(\psi_{\nu}^{*}\right)$. By means of the family of norms $N_{\nu, m}(f)$ $\left(m \in \mathbb{Z}_{+}\right)$we introduce a locally convex topology in $Q\left(\psi_{\nu}^{*}\right)$. We equip $Q\left(\Psi^{*}\right)$ by the topology of inductive limit of spaces $Q\left(\psi_{\nu}^{*}\right)$. 
Lemma 4. Suppose that family $\Phi$ satisfies condition $\left.i_{3}\right)$. Then $Q\left(\Psi^{*}\right)=G\left(\Psi^{*}\right)$.

Proof. It is obvious that if $\nu \in \mathbb{N}$ and $f \in Q\left(\psi_{\nu}^{*}\right)$, then for each $m \in \mathbb{Z}_{+}$we have $\|f\|_{m, \psi_{\nu}^{*}} \leqslant$ $N_{\nu, m}(f)$. Thus, $f \in G_{m}\left(\psi_{\nu}^{*}\right)$. Therefore, if $f \in Q\left(\Psi^{*}\right)$, then $f \in G\left(\Psi^{*}\right)$ and the embedding $J: Q\left(\Psi^{*}\right) \rightarrow G\left(\Psi^{*}\right)$ is continuous.

Let us show that $J$ is surjective. Let $\nu \in \mathbb{N}, m \in \mathbb{N}, f \in G\left(\psi_{\nu}^{*}\right)$. By inequality we get

$$
\sup _{\|x\| \leqslant 1, k \in \mathbb{Z}_{+}} \frac{(1+\|x\|)^{k}\left\|\left(D^{\alpha} f\right)(x)\right\|}{k ! e^{-\psi_{\nu+n}^{*}(k)}} \leqslant \sup _{\|x\| \leqslant 1, k \in \mathbb{Z}_{+}} \frac{e^{a_{\nu+n-1}}\left\|\left(D^{\alpha} f\right)(x)\right\|}{k ! e^{-\psi_{\nu+n-1}^{*}(k)}} .
$$

Since $\lim _{k \rightarrow \infty} \frac{e^{\psi_{\nu+n-1}^{*}(k)}}{k !}=0$, then

$$
\sup _{\|x\| \leqslant 1, k \in \mathbb{Z}_{+}} \frac{(1+\|x\|)^{k}\left\|\left(D^{\alpha} f\right)(x)\right\|}{k ! e^{-\psi_{\nu+n}^{*}(k)}} \leqslant C_{1}(\nu) \sup _{\|x\| \leqslant 1}\left\|\left(D^{\alpha} f\right)(x)\right\|, \alpha \in \mathbb{Z}_{+}^{n} .
$$

for some $C_{1}(\nu)>1$ Since

$$
\left\|\left(D^{\alpha} f\right)(x)\right\| \leqslant\|f\|_{m, \psi_{\nu}^{*}} e^{-\psi_{\nu}^{*}(0)}, \quad x \in \mathbb{R}^{n},
$$

for each $\alpha \in \mathbb{Z}_{+}^{n}$ obeying $\|\alpha\| \leqslant m$, by inequality (9) we obtain that

$$
\max _{\|\alpha\| \leqslant m} \sup _{\|x\| \leqslant 1, k \in \mathbb{Z}_{+}} \frac{(1+\|x\|)^{k}\left\|\left(D^{\alpha} f\right)(x)\right\|}{k ! e^{-\psi_{\nu+n}^{*}(k)}} \leqslant C_{1}(\nu)\|f\|_{m, \psi_{\nu}^{*}} e^{-\psi_{\nu}^{*}(0)} .
$$

For each $\alpha \in \mathbb{Z}_{+}^{n}$

$$
\sup _{\|x\|>1, k \in \mathbb{Z}_{+}} \frac{(1+\|x\|)^{k}\left\|\left(D^{\alpha} f\right)(x)\right\|}{k ! e^{-\psi_{\nu+n}^{*}(k)}} \leqslant \sup _{\|x\|>1, \beta \in \mathbb{Z}_{+}^{n}} \frac{(2 n)^{\|\beta\|}\left\|x^{\beta}\left(D^{\alpha} f\right)(x)\right\|}{\|\beta\| ! e^{-\psi_{\nu+n}^{*}(\|\beta\|)}} .
$$

Employing inequality (1), for some $C_{2}(\nu)>1$ and each $\alpha \in \mathbb{Z}_{+}^{n},\|\alpha\| \leqslant m$ we have

$$
\sup _{\substack{\|x\|>1, k \in \mathbb{Z}_{+}}} \frac{(1+\|x\|)^{k}\left\|\left(D^{\alpha} f\right)(x)\right\|}{k ! e^{-\psi_{\nu+n}^{*}(k)}} \leqslant \sup _{\substack{\|x\|>1, \beta \in \mathbb{Z}_{+}^{n}}} \frac{C_{2}(\nu)\left\|x^{\beta}\left(D^{\alpha} f\right)(x)\right\|}{\|\beta\| ! e^{-\psi_{\nu}^{*}(\|\beta\|)}} \leqslant C_{2}(\nu)\|f\|_{m, \psi_{\nu}^{*}} .
$$

By (10) it implies that for each $m \in \mathbb{Z}_{+}$

$$
N_{\nu+n, m}(f) \leqslant C(\nu)\|f\|_{m, \psi_{\nu}^{*}}, \quad f \in G\left(\psi_{\nu}^{*}\right),
$$

where $C(\nu)=\max \left(C_{1}(\nu) e^{-\psi_{\nu}^{*}(0)}, C_{2}(\nu)\right)$. Therefore, $f \in Q\left(\psi_{\nu+n}^{*}\right)$. Thus, if $f \in G\left(\Psi^{*}\right)$, then $f \in Q\left(\Psi^{*}\right)$. We note that it follows easily from (11) that the inverse mapping $J^{-1}$ is continuous. Hence, we finally get $Q\left(\psi^{*}\right)=G\left(\psi^{*}\right)$.

4.2. Proof of Theorem 3. Let us show first that the linear mapping $\mathcal{F}: f \in E(\Phi) \rightarrow \hat{f}$ acts from $E(\Phi)$ into $G\left(\Psi^{*}\right)$ and is continuous. Let $\nu \in \mathbb{N}$ and $f \in E\left(\varphi_{\nu}\right)$. Employing inequality

$$
x^{\beta}\left(D^{\alpha} \hat{f}\right)(x)=x^{\beta} \int_{\mathbb{R}^{n}} f(\zeta)(-i \zeta)^{\alpha} e^{-i\langle x, \zeta\rangle} d \xi, \quad \zeta=\xi+i \eta,
$$

valid for each $\alpha, \beta \in \mathbb{Z}_{+}^{n}, x, \eta \in \mathbb{R}^{n}$, we obtain that

$$
\left\|x^{\beta}\left(D^{\alpha} \hat{f}\right)(x)\right\| \leqslant \int_{\mathbb{R}^{n}} \frac{\|f(\zeta)\|(1+\|\zeta\|)^{n+\|\alpha\|+1} e^{\langle x, \eta\rangle}\|x\|^{\|\beta\|}}{(1+\|\xi\|)^{n+1}} d \xi .
$$

If $\|\beta\|=0$, then by inequality (12) we have (as $\eta=0$ )

$$
\left\|\left(D^{\alpha} \hat{f}\right)(x)\right\| \leqslant s_{n}(1) e^{\varphi_{\nu}(0)} p_{\nu, n+\|\alpha\|+1}(f) .
$$


If $\|\beta\|>0, x \neq 0$, letting $\eta=-\frac{x t}{\|x\|}$ with $t>0$, by (12) we obtain that

$$
\begin{aligned}
\left\|x^{\beta}\left(D^{\alpha} \hat{f}\right)(x)\right\| & \leqslant s_{n}(1) p_{\nu, n+\|\alpha\|+1}(f) e^{-t\|x\|} e^{\varphi_{\nu}(t)}\|x\|^{\|\beta\|} \\
& \leqslant s_{n}(1) p_{\nu, n+\|\alpha\|+1}(f) e^{\sup (-t r+\|\beta\| \ln r)} e^{\varphi_{\nu}(t)} \\
& =s_{n}(1) p_{\nu, n+\|\alpha\|+1}(f) e^{\|\beta\| \ln \|\beta\|-\|\beta\|-\|\beta\| \ln t} e^{\varphi_{\nu}(t)} .
\end{aligned}
$$

Since for each $k \in \mathbb{Z}_{+}$

$$
\begin{aligned}
\inf _{t>0}\left(-k \ln t+\varphi_{\nu}(t)\right) & =-\sup _{t>0}\left(k \ln t-\varphi_{\nu}(t)\right) \leqslant-\sup _{t \geqslant 1}\left(k \ln t-\varphi_{\nu}(t)\right) \\
& =-\sup _{u \geqslant 0}\left(k u-\psi_{\nu}(u)\right)=-\psi_{\nu}^{*}(k),
\end{aligned}
$$

by the previous estimate we obtain

$$
\left\|x^{\beta}\left(D^{\alpha} \hat{f}\right)(x)\right\| \leqslant s_{n}(1) p_{\nu, n+\|\alpha\|+1}(f) e^{\|\beta\| \ln \|\beta\|-\|\beta\|} e^{-\psi_{\nu}^{*}(\|\beta\|)} .
$$

If $\|\beta\|>0$ and $x=0$, then $x^{\beta}\left(D^{\alpha} \hat{f}\right)(x)=0$. By inequalities (13) and (14) it follows that

$$
\left\|x^{\beta}\left(D^{\alpha} \hat{f}\right)(x)\right\| \leqslant s_{n}(1) p_{\nu, n+\|\alpha\|+1}(f)\|\beta\| ! e^{-\psi_{\nu}^{*}(\|\beta\|)}
$$

for each $\alpha, \beta \in \mathbb{Z}_{+}^{n}, x \in \mathbb{R}^{n}$. Thus,

$$
\max _{\|\alpha\| \leqslant m} \sup _{x \in \mathbb{R}^{n}, \beta \in \mathbb{Z}_{+}^{n}} \frac{\left\|x^{\beta}\left(D^{\alpha} \hat{f}\right)(x)\right\|}{\|\beta\| ! e^{-\psi_{\nu}^{*}(\|\beta\|)}} \leqslant s_{n}(1) p_{\nu, n+m+1}(f), \quad f \in E\left(\varphi_{\nu}\right),
$$

for each $m \in \mathbb{Z}_{+}$. In other words, $\|\hat{f}\|_{m, \psi_{\nu}^{*}} \leqslant s_{n}(1) p_{\nu, n+m+1}(f), f \in E\left(\varphi_{\nu}\right)$. It means that mapping $\mathcal{F}$ acts from $E(\Phi)$ into $G\left(\Psi^{*}\right)$ and is continuous.

Let us show that $\mathcal{F}$ is surjective. Let $g \in G\left(\Psi^{*}\right)$. Then $g \in G\left(\psi_{\nu}^{*}\right)$ for some $\nu \in \mathbb{N}$. In accordance with the proof of Lemma $4, g \in Q\left(\psi_{\nu+n}^{*}\right)$. Then

$$
(1+\|x\|)^{k}\left\|\left(D^{\alpha} g\right)(x)\right\| \leqslant N_{\nu+n,\|\alpha\|}(g) k ! e^{-\psi_{\nu+n}^{*}(k)}, \quad k \in \mathbb{Z}_{+}, \quad \alpha \in \mathbb{Z}_{+}^{n}, \quad x \in \mathbb{R}^{n} .
$$

Let

$$
f(\xi)=\frac{1}{(2 \pi)^{n}} \int_{\mathbb{R}^{n}} g(x) e^{i\langle x, \xi\rangle} d x, \quad \xi \in \mathbb{R}^{n} .
$$

Let $\alpha=\left(\alpha_{1}, \ldots, \alpha_{n}\right), \beta=\left(\beta_{1}, \ldots, \beta_{n}\right) \in \mathbb{Z}_{+}^{n}, \xi \in \mathbb{R}^{n}$. We denote $\gamma_{s}=\min \left(\alpha_{s}, \beta_{s}\right)$ for $s=$ $1, \ldots, n, \gamma=\left(\gamma_{1}, \ldots, \gamma_{n}\right)$. Employing the identity

$$
(i \xi)^{\beta}\left(D^{\alpha} f\right)(\xi)=\frac{(-1)^{\|\beta\|}}{(2 \pi)^{n}} \int_{\mathbb{R}^{n}} \sum_{j \in \mathbb{Z}_{+}^{n}: j \leqslant \gamma} C_{\beta}^{j}\left(D^{\beta-j} g\right)(x)\left(D^{j}(i x)^{\alpha}\right) e^{i\langle x, \xi\rangle} d x
$$

and inequality (15), we estimate the absolute value of $\xi^{\beta}\left(D^{\alpha} f\right)(\xi)$. We have

$$
\begin{aligned}
\left\|\xi^{\beta}\left(D^{\alpha} f\right)(\xi)\right\| & \leqslant \frac{1}{(2 \pi)^{n}} \sum_{j \in \mathbb{Z}_{+}^{n}: j \leqslant \gamma} C_{\beta}^{j} \int_{\mathbb{R}^{n}}\left\|\left(D^{\beta-j} g\right)(x)\right\| \frac{\alpha !}{(\alpha-j) !}\|x\|^{\|\alpha-j\|} d x \\
& \leqslant \frac{1}{(2 \pi)^{n}} \sum_{j \in \mathbb{Z}_{+}^{n}: j \leqslant \gamma} C_{\beta}^{j} \frac{\alpha !}{(\alpha-j) !} \int_{\mathbb{R}^{n}}\left\|\left(D^{\beta-j} g\right)(x)\right\|(1+\|x\|)^{\|\alpha-j\|+n+1} \frac{d x}{(1+\|x\|)^{n+1}} \\
& \leqslant \frac{s_{n}(1)}{(2 \pi)^{n}} \sum_{j \in \mathbb{Z}_{+}^{n}: j \leqslant \gamma} C_{\beta}^{j} \frac{\alpha !}{(\alpha-j) !} \frac{N_{\nu+n,\|\beta\|}(g)(\|\alpha\|-\|j\|+n+1) !}{e^{\psi_{\nu+n}^{*}(\|\alpha\|-\|j\|+n+1)}} \\
& \leqslant \frac{s_{n}(1) N_{\nu+n,\|\beta\|}(g) \alpha !}{(2 \pi)^{n}} \sum_{j \in \mathbb{Z}_{+}^{n}: j \leqslant \gamma} C_{\beta}^{j} \frac{(\|\alpha\|-\|j\|+n+1) !}{(\alpha-j) !} e^{-\psi_{\nu+n}^{*}(\|\alpha\|-\|j\|) .}
\end{aligned}
$$


We note that condition $i_{4}$ ) for $\Phi$ implies that

$$
2 \psi_{k}(x) \leqslant \psi_{k+1}\left(x+b_{k}\right)+l_{k}, \quad x \geqslant 0,
$$

for each $k \in \mathbb{N}$, where $b_{k}=\ln h_{k}$. Then in accordance with Lemma 2 , for some $A_{k}>0$

$$
\psi_{k+1}^{*}(x+y) \leqslant \psi_{k}^{*}(x)+\psi_{k}^{*}(y)+b_{k}(x+y)+A_{k}, \quad x, y \geqslant 0 .
$$

Employing inequality (16) and letting $c_{1}=\frac{s_{n}(1) e^{A_{\nu+n}}}{(2 \pi)^{n}}$, we get

$$
\left\|\xi^{\beta}\left(D^{\alpha} f\right)(\xi)\right\| \leqslant \frac{c_{1} e^{b_{\nu+n}\|\alpha\|} N_{\nu+n,\|\beta\|}(g) \alpha !}{e^{\psi_{\nu+n+1}^{*}(\|\alpha\|)}} \sum_{\substack{j \in \mathbb{Z}_{+}^{n}: \\ j \leqslant \gamma}} \frac{C_{\beta}^{j}(\|\alpha\|-\|j\|+n+1) ! e^{\psi_{\nu+n}^{*}(\|j\|)}}{(\alpha-j) !} .
$$

We observe that $\left(m_{1}+m_{2}\right) ! \leqslant e^{m_{1}+m_{2}} m_{1} ! m_{2} !$ for $m_{1}, m_{2} \in \mathbb{Z}_{+}$. It yields that

$$
\left(m_{1}+\cdots+m_{n}\right) ! \leqslant e^{(n-1)\left(m_{1}+\cdots+m_{n}\right)} m_{1} ! \cdot \ldots \cdot m_{n} !, \quad m_{1}, \ldots, m_{n} \in \mathbb{Z}_{+} .
$$

Employing this inequality and letting $c_{2}=c_{1} e^{n+1}(n+1)$ !, we obtain

$$
\left\|\xi^{\beta}\left(D^{\alpha} f\right)(\xi)\right\| \leqslant \frac{c_{2} e^{b_{\nu+n}\|\alpha\|} N_{\nu+n,\|\beta\|}(g) \alpha !}{e^{\psi_{\nu+n+1}^{*}(\|\alpha\|)}} \sum_{j \in \mathbb{Z}_{+}^{n}: j \leqslant \gamma} C_{\beta}^{j} \frac{e^{\|\alpha\|-\|j\|}(\|\alpha\|-\|j\|) ! e^{\psi_{\nu+n}^{*}(\|j\|)}}{(\alpha-j) !} .
$$

Using inequality (17) once again, we get

$$
\left\|\xi^{\beta}\left(D^{\alpha} f\right)(\xi)\right\| \leqslant \frac{c_{2} e^{b_{\nu+n}\|\alpha\|} N_{\nu+n,\|\beta\|}(g) \alpha !}{e^{\psi_{\nu+n+1}^{*}(\|\alpha\|)}} \sum_{j \in \mathbb{Z}_{+}^{n}: j \leqslant \gamma} C_{\beta}^{j} e^{n(\|\alpha\|-\|j\|)} e^{\psi_{\nu+n}^{*}(\|j\|)} .
$$

Thus,

$$
\left\|\xi^{\beta}\left(D^{\alpha} f\right)(\xi)\right\| \leqslant \frac{c_{2} \beta ! e^{\left(b_{\nu+n}+n\right)\|\alpha\|} N_{\nu+n,\|\beta\|}(g) \alpha !}{e^{\psi_{\nu+n+1}^{*}(\|\alpha\|)}} \sum_{j \in \mathbb{Z}_{+}^{n}: j \leqslant \gamma} \frac{e^{\psi_{\nu+n}^{*}(\|j\|)}}{e^{n\|j\| j} \cdot .} .
$$

Applying inequality (17) one more time and letting $c_{3}=c_{2} \beta ! \sum_{\|j\| \geqslant 0} \frac{e^{\psi_{\nu+n}^{*}(\|j\|)}}{\|j\| !}$ (series $\sum_{\|j\| \geqslant 0} \frac{e^{\psi_{\nu+n}^{*}(\|j\|)}}{\|j !\|}$ converges (cf. Corollary 1)), we obtain

$$
\left\|\xi^{\beta}\left(D^{\alpha} f\right)(\xi)\right\| \leqslant c_{3} e^{\left(b_{\nu+n}+n\right)\|\alpha\|} N_{\nu+n,\|\beta\|}(g) \alpha ! e^{-\psi_{\nu+n+1}^{*}(\|\alpha\|)} .
$$

Hence, in view of inequality (1), we find a natural number $s=s(\nu, n)>n+1$ and a constant $c_{4}>0$ (depending on $\nu, n$, and $\beta$ ) such that

$$
\left\|\xi^{\beta}\left(D^{\alpha} f\right)(\xi)\right\| \leqslant c_{4} N_{\nu+n,\|\beta\|}(g) \alpha ! e^{-\psi_{\nu+s}^{*}(\|\alpha\|)} .
$$

Thus, if $m \in \mathbb{Z}_{+}$, the last inequality implies

$$
(1+\|\xi\|)^{m}\left\|\left(D^{\alpha} f\right)(\xi)\right\| \leqslant c_{5} N_{\nu+n, m}(g) \alpha ! e^{-\psi_{\nu+s}^{*}(\|\alpha\|)}, \alpha \in \mathbb{Z}_{+}^{n}, \xi \in \mathbb{R}^{n},
$$

where $c_{5}>0$ is a constant depending on $\nu, n$, and $m$. By Theorem $2, f$ can be holomorphically continued to an entire function $F_{f}$ in $E(\Phi)$. It is obvious that $g=\mathcal{F}\left(F_{f}\right)$. The proof of Theorem 2 (see inequalities (3) and (8)) shows that there exists a constant $c_{6}>0$ (depending on $\nu, n$, and $m$ ) such that

$$
(1+\|z\|)^{m}\left\|F_{f}(z)\right\| \leqslant c_{6} N_{\nu+n, m}(g) e^{\varphi_{\nu+s+3}(\|\operatorname{Im} z\|)}
$$

for $z \in \mathbb{C}^{n}$. Therefore, $p_{\nu+s+3, m}\left(F_{f}\right) \leqslant c_{6} N_{\nu+n, m}(g)$. In view of inequality (11) we obtain $p_{\nu+s+3, m}\left(F_{f}\right) \leqslant c_{7}\|g\|_{m, \psi_{\nu}^{*}}, g \in G\left(\psi_{\nu}^{*}\right)$, where $c_{7}>0$ is a constant depending on $\nu, n$, and $m$. It follows the continuity of the inverse mapping $\mathcal{F}^{-1}$.

Thus, we have proven that Fourier transform makes an isomorphism between the spaces $E(\Phi)$ and $G\left(\Psi^{*}\right)$.

4.3. On an approach for constructing family $\Phi$. Let $U=\left\{u_{\nu}\right\}_{\nu=1}^{\infty}$ be a family of non-decreasing convex functions $u_{\nu}$ on $[0, \infty)$ such that for each $\nu$ : 
1. $\lim _{x \rightarrow+\infty} \frac{u_{\nu}(x)}{x}=+\infty$;

2. given $M>0$, there exists a constant $A(M, \nu)>0$ such that

$$
u_{\nu}(x) \leqslant x \ln \frac{x}{M}+A(M, \nu), \quad x>0 ;
$$

3. given $A>0$, there exists a constant $K(\nu, A)>0$ such that

$$
u_{\nu+1}(x+A) \leqslant u_{\nu}(x)+K(\nu, A), \quad x \geqslant 0 ;
$$

4. there exists a constant $a_{\nu}>0$ such that

$$
u_{\nu}(x)-u_{\nu+1}(x) \geqslant x \ln 2-a_{\nu}, \quad x \geqslant 0 ;
$$

5. there exists a constant $A_{\nu}>0$ such that

$$
u_{\nu+1}(2 x) \leqslant 2 u_{\nu}(x)+x \ln 4+A_{\nu}, \quad x \geqslant 0 .
$$

Suppose that family $\Phi$ consists of functions $\varphi_{\nu}$ defined on $[0, \infty)$ by the rule: $\varphi_{\nu}(x)=$ $u_{\nu}^{*}(\ln (1+x)), x \geqslant 0(\nu \in \mathbb{N})$. It is clear that functions $\varphi_{\nu}$ do not decrease and are continuous on $[0, \infty)$. First two conditions follow that the constructed family satisfies condition $\left.i_{1}\right)$. The third condition ensures condition $i_{2}$ ). By the forth condition it is easy to get that for each $\nu \in \mathbb{N}$

$$
u_{\nu}^{*}(t+\ln 2)-u_{\nu+1}^{*}(t) \leqslant a_{\nu}, \quad t \geqslant 0 .
$$

Hence, for each $x \geqslant 0$ we have

$$
\begin{aligned}
\varphi_{\nu}(2 x)=u_{\nu}^{*}(\ln (1+2 x)) & \leqslant u_{\nu}^{*}(\ln (2(1+x)))=u_{\nu}^{*}(\ln 2+\ln (x+1)) \\
& \leqslant u_{\nu+1}^{*}(\ln (x+1))+a_{\nu}=\varphi_{\nu+1}(x)+a_{\nu} .
\end{aligned}
$$

Thus, family $\Phi$ satisfies condition $i_{3}$ ). Condition $i_{4}$ ) holds for $\Phi$, too. While checking it, we make use of the following simple statement.

Proposition 2. Suppose that $u, v \in \mathcal{B}$ and there exist numbers $\tau>0$ and $A>0$ such that

$$
v(2 x) \leqslant 2 u(x)+2 \tau x+A, \quad x, y \geqslant 0 .
$$

Then

$$
2 u^{*}(x) \leqslant v^{*}(x+\tau)+A, \quad x \geqslant 0 .
$$

Proof. For each $x \geqslant 0$ we have

$$
\begin{aligned}
2 u^{*}(x) & =\sup _{\xi \geqslant 0}(2 x \xi-2 u(\xi)) \leqslant \sup _{\xi \geqslant 0}(2 x \xi-v(2 \xi)+2 \tau \xi)+A \\
& =\sup _{\xi \geqslant 0}((x+\tau) t-v(t))+A=v^{*}(x+\tau)+A .
\end{aligned}
$$

Returning back to checking condition $i_{4}$ ) for $\Phi$ and employing fifth condition for $U$ and Proposition 2, for each $\nu \in \mathbb{N}$ and $x \geqslant 0$ we have

$$
\begin{aligned}
2 \varphi_{\nu}(x) & =2 u_{\nu}^{*}(\ln (1+x)) \leqslant u_{\nu+1}^{*}(\ln (1+x)+\ln 2)+A_{\nu} \\
& =u_{\nu+1}^{*}\left(\ln ((2 x+1)+1)+A_{\nu}=\varphi_{\nu+1}(2 x+1)+A_{\nu} .\right.
\end{aligned}
$$

It follows that condition $i_{4}$ ) holds true, at that, as $h_{\nu}$ we can take any number greater than 2 .

Thus, family $\Phi$ satisfies the hypothesis of Theorem 3. Thus, Fourier transform makes an isomorphism between spaces $E(\Phi)$ and $G(U)$. 


\section{Special CASE OF FAmily $\Phi$}

In the proof of Theorem 4 we shall make use of the following three lemmata.

Lemma 5. Let $g \in \mathcal{B}$. Then for each $\delta>0$

$$
\lim _{x \rightarrow+\infty} \frac{g^{*}((1+\delta) x)-g^{*}(x)}{x}=+\infty .
$$

Proof. Let $\delta>0$ be arbitrary. For $x>0$ by $\xi(x)$ we denote the point at which the function $u_{x}(\xi)=x \xi-g(\xi)$ attains the supremum over the set $[0, \infty)$. We note that $\xi(x) \rightarrow+\infty$ as $x \rightarrow+\infty$. Otherwise there exists a number $M>0$ and a sequence $\left(x_{j}\right)_{j=1}^{\infty}$ of positive numbers $x_{j}$ tending to $+\infty$ such that $\xi\left(x_{j}\right) \leqslant M$. Then $g^{*}\left(x_{j}\right)=x_{j} \xi\left(x_{j}\right)-g\left(\xi\left(x_{j}\right)\right)$. But it contradicts to the identity $\lim _{x \rightarrow+\infty} \frac{g^{*}(x)}{x}=+\infty$. Hence, $\lim _{x \rightarrow+\infty} \xi(x)=+\infty$. Together with inequality

$$
g^{*}((1+\delta) x)-g^{*}(x) \geqslant(1+\delta) x \xi(x)-g(\xi(x))-x \xi(x)+g(\xi(x))=\delta x \xi(x), \quad x>0,
$$

it completes the proof.

The next statement follows easily from the results by S.V. Popenov (see Lemma 4 in [9]) and this is why we do not provide its proof.

Lemma 6. Let $u \in V$. There exists a constant $K>0$ (depending on $u$ ) such that

$$
(u[e])^{*}(t)+\left(u^{*}[e]\right)^{*}(t) \geqslant t \ln t-t-K, \quad t>0 .
$$

The next lemma was proven in [8].

Lemma 7. Let $u \in \mathcal{B}$. Then

$$
(u[e])^{*}(x)+\left(u^{*}[e]\right)^{*}(x) \leqslant x \ln x-x, \quad x>0 .
$$

Proof of Theorem 4. Let $\nu \in \mathbb{N}, f \in G\left(\psi_{\nu}^{*}\right)$. We fix $m \in \mathbb{Z}_{+}$. Since $f \in Q\left(\psi_{\nu+n}^{*}\right)$ (see the proof of Lemma 4), then for each $k \in \mathbb{Z}_{+}, \alpha \in \mathbb{Z}_{+}^{n},\|\alpha\| \leqslant m, x \in \mathbb{R}^{n}$,

$$
\left\|\left(D^{\alpha} f\right)(x)\right\| \leqslant \frac{N_{\nu+n, m}(f) k ! e^{-\psi_{\nu+n}^{*}(k)}}{(1+\|x\|)^{k}} .
$$

Taking into consideration that $j !<\frac{3 j^{j+1}}{e^{j}}$ for $j \in \mathbb{N}$ and employing inequality (16) and the non-decreasing of function $\psi_{\nu+n}^{*}$, we obtain

$$
\frac{k ! e^{-\psi_{\nu+n}^{*}(k)}}{\mu^{k}} \leqslant \frac{3 k^{k+1} e^{-\psi_{\nu+n}^{*}(k)}}{e^{k} \mu^{k}} \leqslant \frac{3 \mu t^{t+1} e^{-\psi_{\nu+n+1}^{*}(t)+\psi_{\nu+n}^{*}(1)+b_{\nu+n} t+A_{\nu+n}}}{e^{t} \mu^{t}}
$$

for each $k \in \mathbb{N}, t \in[k, k+1)$ and $\mu \geqslant 1$.

We employ (1) to obtain

$$
\frac{k ! e^{-\psi_{\nu+n}^{*}(k)}}{\mu^{k}} \leqslant C_{1} \mu e^{(t+1) \ln t-\psi_{\nu+s}^{*}(t)-t \ln e \mu+\left(b_{\nu+n}-s \ln 2\right) t},
$$

where $s \geqslant n+2$ is a natural number and $C_{1}$ is a positive constant depending on $\nu, n$, and $s$. We choose $s \in \mathbb{N}$ so that $s \ln 2>b_{\nu+n}$. We find a constant $C_{2}>0$ (depending on $\nu, n$, and chosen $s$ ) such that

$$
\frac{k ! e^{-\psi_{\nu+n}^{*}(k)}}{\mu^{k}} \leqslant C_{2} \mu e^{t \ln t-\psi_{\nu+s}^{*}(t)-t \ln \mu} .
$$

Now by Lemma 6 we obtain

$$
\frac{k ! e^{-\psi_{\nu+n}^{*}(k)}}{\mu^{k}} \leqslant C_{3} \mu e^{\left(\varphi_{\nu+s}^{*}[e]\right)^{*}(t)-t \ln \mu}
$$


where $C_{3}$ is a positive constant depending on $\nu, n$, and chosen $s$. It follows that

$$
\inf _{k \in \mathbb{N}} \frac{k ! e^{-\psi_{\nu+n}^{*}(k)}}{\mu^{k}} \leqslant C_{3} \mu e^{\inf _{t \geqslant 1}\left(\varphi_{\nu+s}^{*}[e]\right)^{*}(t)-t \ln \mu} .
$$

It is obvious that

$$
\begin{aligned}
\inf _{t \geqslant 1}\left(\left(\varphi_{\nu+s}^{*}[e]\right)^{*}(t)-t \ln \mu\right) & \leqslant-\ln \mu+\left(\varphi_{\nu+s}^{*}[e]\right)^{*}(1) \\
\inf _{0<t \leqslant 1}\left(\left(\varphi_{\nu+s}^{*}[e]\right)^{*}(t)-t \ln \mu\right) & \geqslant-\ln \mu+\left(\varphi_{\nu+s}^{*}[e]\right)^{*}(0) .
\end{aligned}
$$

Thus,

$$
\inf _{t \geqslant 1}\left(\left(\varphi_{\nu+s}^{*}[e]\right)^{*}(t)-t \ln \mu\right) \leqslant \inf _{0<t \leqslant 1}\left(\left(\varphi_{\nu+s}^{*}[e]\right)^{*}(t)-t \ln \mu\right)+\left(\varphi_{\nu+s}^{*}[e]\right)^{*}(1)-\left(\varphi_{\nu+s}^{*}[e]\right)^{*}(0) .
$$

Denoting $\left(\varphi_{\nu+s}^{*}[e]\right)^{*}(1)-\left(\varphi_{\nu+s}^{*}[e]\right)^{*}(0)$ by $m_{\nu}$, we get

$$
\inf _{t \geqslant 1}\left(\left(\varphi_{\nu+s}^{*}[e]\right)^{*}(t)-t \ln \mu\right) \leqslant \inf _{t>0}\left(\left(\varphi_{\nu+s}^{*}[e]\right)^{*}(t)-t \ln \mu\right)+m_{\nu}
$$

Returning back to (19), we obtain

$$
\inf _{k \in \mathbb{N}} \frac{k ! e^{-\psi_{\nu+n}^{*}(k)}}{\mu^{k}} \leqslant C_{3} e^{m_{\nu}} \mu e^{\inf _{>0}\left(\left(\varphi_{\nu+s}^{*}[e]\right)^{*}(t)-t \ln \mu\right)} .
$$

For each $j \in \mathbb{N}$ we choose $\theta_{j} \in V_{\varphi_{j}^{*}[e]}$. Then

$$
\begin{aligned}
& \left\|\theta_{j}(\xi)-\varphi_{j}^{*}[e](\xi)\right\| \leqslant r_{j}, \quad \xi \geqslant 0 ; \\
& \left\|\theta_{j}^{*}(\xi)-\left(\varphi_{j}^{*}[e]\right)^{*}(\xi)\right\| \leqslant r_{j}, \quad \xi \geqslant 0,
\end{aligned}
$$

where $r_{j}$ is a positive constant depending on $\varphi_{j}^{*}[e]$ and $\theta_{j}$. By (20) and (22) we get

$$
\inf _{k \in \mathbb{N}} \frac{k ! e^{-\psi_{\nu+n}^{*}(k)}}{\mu^{k}} \leqslant C_{4} \mu e^{\inf _{t>0}\left(\theta_{\nu+s}^{*}(t)-t \ln \mu\right)},
$$

where $C_{4}=C_{3} e^{m_{\nu}+r_{\nu+s}}$. Employing the formula for inversion of Young transform [10], we obtain

$$
\inf _{k \in \mathbb{N}} \frac{k ! e^{-\psi_{\nu+n}^{*}(k)}}{\mu^{k}} \leqslant C_{4} \mu e^{-\theta_{\nu+s}(\ln \mu)} .
$$

By inequality (21) it yields

$$
\inf _{k \in \mathbb{N}} \frac{k ! e^{-\psi_{\nu+n}^{*}(k)}}{\mu^{k}} \leqslant C_{5} \mu e^{-\varphi_{\nu+s}^{*}[e](\ln \mu)}
$$

where $C_{5}=C_{\nu, 4} e^{r_{\nu+s}}$. Thus,

$$
\inf _{k \in \mathbb{N}} \frac{k ! e^{-\psi_{\nu+n}^{*}(k)}}{\mu^{k}} \leqslant C_{5} \mu e^{-\varphi_{\nu+s}^{*}(\mu)} .
$$

Employing this inequality and the non-decreasing of $\varphi_{\nu+s}^{*}$, we have

$$
\inf _{k \in \mathbb{N}} \frac{k ! e^{-\psi_{\nu+n}^{*}(k)}}{(1+\|x\|)^{k}} \leqslant C_{5}(1+\|x\|) e^{-\varphi_{\nu+s}^{*}(\|x\|)}, x \in \mathbb{R}^{n} .
$$

We observe that using condition $i_{3}$ ) for $\Phi$, it is easy to show that for each $j \in \mathbb{N}$

$$
\varphi_{j+1}^{*}(\xi) \leqslant \varphi_{j}^{*}\left(\frac{\xi}{2}\right)+a_{j}, \xi \geqslant 0 .
$$

Therefore,

$$
\varphi_{j}^{*}(\xi)-\varphi_{j+1}^{*}(\xi) \geqslant \varphi_{j}^{*}(\xi)-\varphi_{j}^{*}\left(\frac{\xi}{2}\right)-a_{j}, \quad \xi \geqslant 0
$$


By Lemma 5 it implies

$$
\lim _{x \rightarrow+\infty} \frac{\varphi_{j}^{*}(\xi)-\varphi_{j+1}^{*}(\xi)}{\xi}=+\infty
$$

Returning back to (23), by means of (25) we obtain

$$
\inf _{k \in \mathbb{N}} \frac{k ! e^{-\psi_{\nu+n}^{*}(k)}}{(1+\|x\|)^{k}} \leqslant C_{6} e^{-\varphi_{\nu+s+1}^{*}(\|x\|)}, x \in \mathbb{R}^{n},
$$

where $C_{6}$ is a positive number. By inequality (18) it follows that

$$
\left\|\left(D^{\alpha} f\right)(x)\right\| \leqslant C_{6} N_{\nu+n, m}(f) e^{-\varphi_{\nu+s+1}^{*}(\|x\|)}
$$

for each $\alpha \in \mathbb{Z}_{+}^{n}$ obeying $\|\alpha\| \leqslant m$. It means that $q_{m, \nu+s+1}(f) \leqslant C_{6} N_{\nu+n, m}(f), f \in G\left(\psi_{\nu}^{*}\right)$. In view of inequality (11), we have

$$
q_{m, \nu+s+1}(f) \leqslant C_{7}\|f\|_{m, \psi_{\nu}^{*}}, \quad f \in G\left(\psi_{\nu}^{*}\right),
$$

where $C_{7}$ is a positive number depending on $\nu$. It follows that the identity mapping $I$ acts from $G\left(\Psi^{*}\right)$ into $G S\left(\Phi^{*}\right)$ and is continuous.

Let us show that $I$ is surjective. Let $f \in G S\left(\Phi^{*}\right)$. Then $f \in G S\left(\varphi_{\nu}^{*}\right)$ for some $\nu \in \mathbb{N}$. We fix $m \in \mathbb{Z}_{+}$. Let $x \in \mathbb{R}^{n}, \alpha \in \mathbb{Z}_{+}^{n}$, at that, $\|\alpha\| \leqslant m$. Then

$$
\left\|\left(D^{\alpha} f\right)(x)\right\| \leqslant q_{m, \nu}(f) e^{-\varphi_{\nu}^{*}(\|x\|)} .
$$

Employing inequality (24), by (27) we obtain

$$
\left\|\left(D^{\alpha} f\right)(x)\right\| \leqslant e^{a_{\nu}} q_{m, \nu}(f) e^{-\varphi_{\nu+1}^{*}(2\|x\|)} .
$$

It is obvious that there exists a constant $M_{\nu}>1$ such that

$$
\left\|\left(D^{\alpha} f\right)(x)\right\| \leqslant M_{\nu} q_{m, \nu}(f) e^{-\varphi_{\nu+1}^{*}(\|x\|+1)} .
$$

In other words,

$$
\left\|\left(D^{\alpha} f\right)(x)\right\| \leqslant M_{\nu} q_{m, \nu}(f) e^{-\varphi_{\nu+1}^{*}[e](\ln (\|x\|+1))} .
$$

It follows that

$$
\left\|\left(D^{\alpha} f\right)(x)\right\| \leqslant M_{\nu} q_{m, \nu}(f) e^{-\sup _{t>0}\left(t \ln (\|x\|+1)-\left(\varphi_{\nu+1}^{*}[e]\right)^{*}(t)\right)} .
$$

Now by Lemma 7 we get

$$
\left\|\left(D^{\alpha} f\right)(x)\right\| \leqslant M_{\nu} q_{m, \nu}(f) e^{-\sup _{t>0}\left(t \ln (e(\|x\|+1))-t \ln t+\psi_{\nu+1}^{*}(t)\right)} .
$$

Therefore, for each $k \in \mathbb{N}$

$$
\left\|\left(D^{\alpha} f\right)(x)\right\| \leqslant M_{\nu} q_{m, \nu}(f) \frac{k^{k} e^{-\psi_{\nu+1}^{*}(k)}}{(e(1+\|x\|))^{k}} .
$$

Hence,

$$
(1+\|x\|)^{k}\left\|\left(D^{\alpha} f\right)(x)\right\| \leqslant M_{\nu} q_{m, \nu}(f) k ! e^{-\psi_{\nu+1}^{*}(k)}, k \in \mathbb{Z}_{+} .
$$

It means that

$$
\|f\|_{m, \psi_{\nu+1}^{*}} \leqslant M_{\nu} q_{m, \nu}(f) .
$$

Since $m \in \mathbb{Z}_{+}$is arbitrary, then $f \in G\left(\psi_{\nu+1}^{*}\right)$. Therefore, $f \in G\left(\Psi^{*}\right)$. It follows from (28) that mapping $I^{-1}$ is continuous. Thus, spaces $G\left(\Psi^{*}\right)$ and $G S\left(\Phi^{*}\right)$ coincide. The proof is complete. 


\section{BIBLIOGRAPHY}

1. B.L. Gurevich. New spaces of test and generalized functions and Cauchy problem for finitedifference systems // Doklady AN SSSR. 99:6, 893-896 (1954). (in Russian).

2. I.M. Gel'fand, G.E. Shilov. Generalized functions. V. 2: Spaces of fundamental and generalized functions. Gos. Izd. Fiz.-Mat. Lit., Moscow (1958). [Academic Press, New Yors (1967).]

3. I.M. Gel'fand, G.E. Shilov. Generalized functions. V. 3: Theory of differential equations. Gos. Izd. Fiz.-Mat. Lit., Moscow (1958). [Academic Press, New York (1967).]

4. J. Chung, S.-Y. Chung, and D. Kim. Characterizations of the Gelfand-Shilov spaces via Fourier transforms // Proc. Amer. Math. Soc. 124:7, 2101-2108 (1996).

5. J. Chung, S.-Y. Chung, and D. Kim. Equivalence of the Gelfand-Shilov spaces // J. Math. Anal. Appl. 203:3, 828-839 (1996).

6. M.I. Musin. On a space of entire functions fast decreasing on a real line // Ufimskij Matem. Zhurn. 4:1, 136-145 (2012). [Ufa Math. J. 4:1, 128-136 (2012).]

7. M.A. Krasnosel'skii, Ya.B. Rutitskii. Convex functions and Orlicz spaces. Gos. Izd. Fiz.-Mat. Lit., Moscow (1958). (in Russian).

8. I.Kh. Musin, S.V. Popenov. On a weighted space of infinitely differentiable functions in $\mathbb{R}^{n} / /$ Ufimskij Matem. Zhurn. 2:3, 52-60 (2010). (in Russian).

9. V.V. Napalkov, S.V. Popenov. On the Laplace transform of functionals in the Bergman weight space of entire functions in $\mathbb{C}^{n} / /$ Doklady RAN. 352:5, 595-597 (1997). [Dokl. Math. 55:1, 110112 (1997).]

10. A.W. Roberts, D.E. Varberg. Convex functions. Academic Press, New York (1973).

Il'dar Khamitovich Musin

Institute of Mathematics CC USC RAS,

Chernyshevsky str., 112,

450077, Ufa, Russia

E-mail: musin@matem.anrb.ru

Marat Il'darovich Musin

Bashkir State University,

Z. Validi str., 32,

450000, Ufa, Russia

E-mail: marat402@gmail.com 